\title{
ONREL \\ Improving Air-Conditioner and Heat Pump Modeling
}

Building America Stakeholders Meeting

\author{
Jon Winkler
}

March 2, 2012

NREL/PR-5500-54361 


\section{Simple Question}

- How do you recommend the most cost-effective $A / C$ ? 


\section{Solution}

Whole-House Simulation Tool

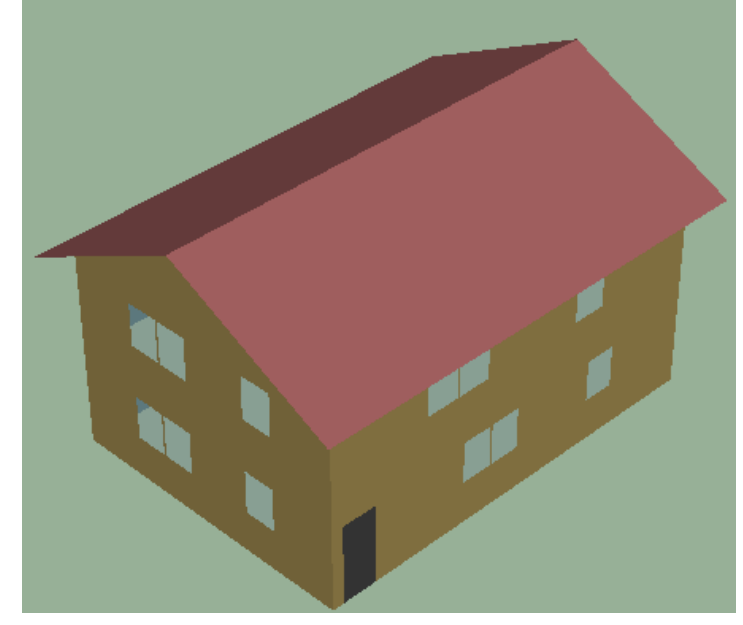

\section{A/C Information}

- SEER 13 - SEER 17

- SEER 14 - SEER 18

- SEER 15 - SEER 21

- SEER 16

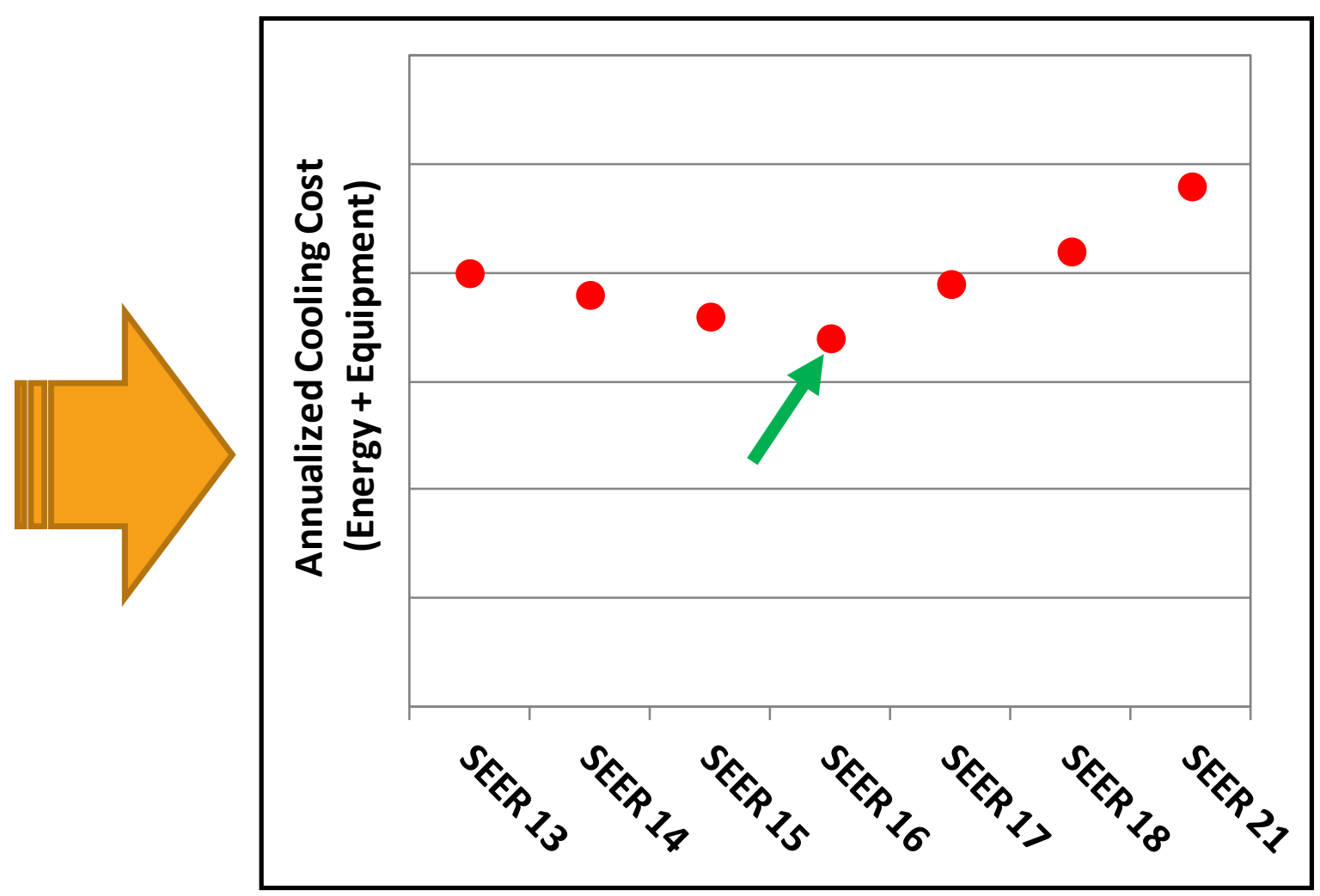




\section{Background}

- Power, capacity and SHR vary with:

- Outdoor temperature

- Entering wetbulb

- Air mass flow rate

- Part load ratio

Power

- How to accurately and easily model A/C performance? 


\section{Background: Model Development}

- $\mathrm{A} / \mathrm{C}$ modeling utilizes two types of input

- Rated values (capacity, efficiency, etc.)

- Performance curves

$$
y=a+b \times E W B+c \times E W B^{2}+d \times O D B+e \times O D B^{2}+f \times O D B \times E W B
$$
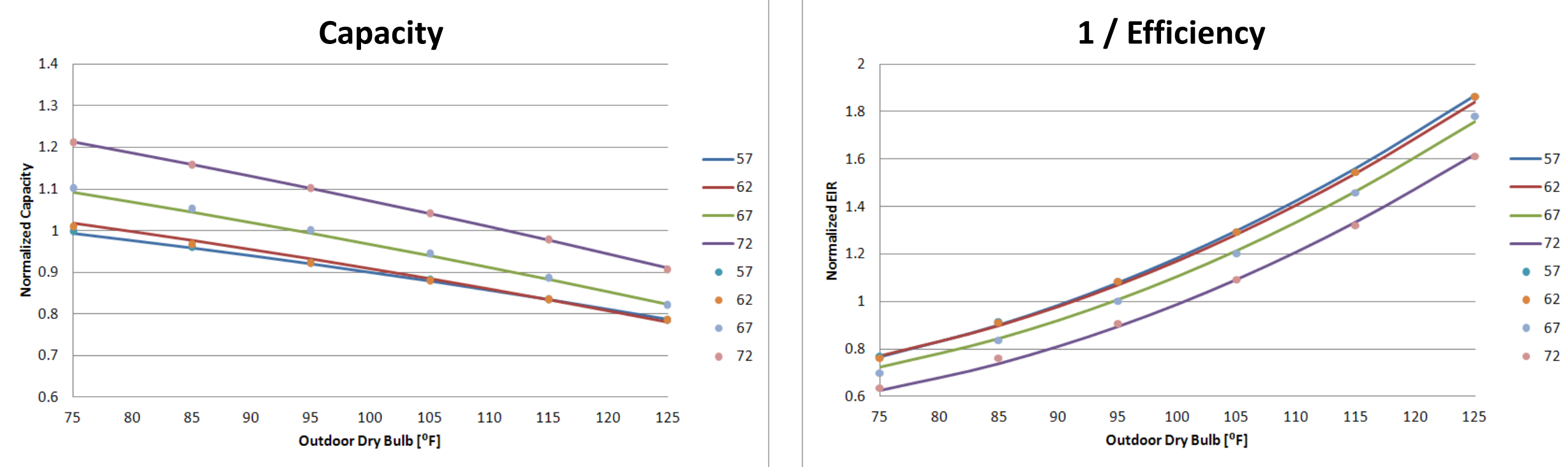


\section{Background: Manufacturer's Data}

\begin{tabular}{|c|c|c|c|c|c|c|c|c|c|c|c|c|c|}
\hline \multirow{2}{*}{\multicolumn{2}{|c|}{ EVAPORATOR AIR }} & & & & & & & \multicolumn{6}{|c|}{ CONDENSER ENTERING AIR TEMPERATURES ${ }^{\circ} \mathrm{F}\left({ }^{\circ} \mathrm{C}\right)$} \\
\hline & & \multicolumn{3}{|c|}{75 (23.9) } & \multicolumn{3}{|c|}{$85(29.4)$} & \multicolumn{3}{|c|}{$95(35)$} & \multicolumn{3}{|c|}{$105(40.6)$} \\
\hline \multirow{2}{*}{ CFM } & \multirow{2}{*}{$\begin{array}{c}\text { EWB } \\
{ }^{\circ} \mathrm{F}\left({ }^{\circ} \mathrm{C}\right)\end{array}$} & \multicolumn{2}{|c|}{ Capacity MBtuh } & \multirow{2}{*}{$\begin{array}{c}\text { Total } \\
\text { System } \\
\text { KW }^{\star \star \star}\end{array}$} & \multicolumn{2}{|c|}{ Capacity MBtuh } & \multirow{2}{*}{$\begin{array}{c}\text { Total } \\
\text { System } \\
\mathrm{KW}^{* *}\end{array}$} & \multicolumn{2}{|c|}{ Capacity MBtuh } & \multirow{2}{*}{$\begin{array}{c}\text { Total } \\
\text { System } \\
\text { KW }^{* \star}\end{array}$} & \multicolumn{2}{|c|}{ Capacity MBtuh } & \multirow{2}{*}{$\begin{array}{r}\text { Total } \\
\text { Systen } \\
\mathrm{KW}^{* \star}\end{array}$} \\
\hline & & Total & Sensł & & Total & Sensł & & Total & Sens‡ & & Total & Senæ‡ & \\
\hline \multirow{5}{*}{875} & $72(22.2)$ & 34.32 & 17.27 & 1.96 & 32.83 & 16.71 & 2.19 & 31.24 & 16.13 & 2.44 & 29.59 & 15.54 & 2.71 \\
\hline & $67(19.4)$ & 31.45 & 21.21 & 1.96 & 30.06 & 20.64 & 2.18 & 28.59 & 20.05 & 2.43 & 27.04 & 19.44 & 2.71 \\
\hline & $63(17.2) \mathrm{tt}$ & 29.35 & 20.58 & 1.96 & 28.04 & 20.00 & 2.18 & 26.66 & 19.40 & 2.43 & 25.19 & 18.78 & 2.70 \\
\hline & $62(16.7)$ & 28.82 & 25.13 & 1.95 & 27.56 & 24.55 & 2.18 & 26.24 & 23.94 & 2.43 & 24.86 & 23.29 & 2.70 \\
\hline & 57 (13.9) & 28.00 & 28.00 & 1.95 & 26.98 & 26.98 & 2.18 & 25.89 & 25.89 & 2.43 & 24.74 & 24.74 & 2.70 \\
\hline \multirow{5}{*}{1000} & $72(22.2)$ & 34.88 & 18.05 & 2.01 & 33.32 & 17.49 & 2.23 & 31.66 & 16.90 & 2.48 & 29.96 & 16.30 & 2.76 \\
\hline & $67(19.4)$ & 31.98 & 22.49 & 2.01 & 30.53 & 21.91 & 2.23 & 29.00 & 21.31 & 2.48 & 27.40 & 20.68 & 2.75 \\
\hline & $63(17.2) \mathrm{tt}$ & 29.88 & 21.78 & 2.00 & 28.51 & 21.19 & 2.23 & 27.07 & 20.58 & 2.48 & 25.55 & 19.95 & 2.75 \\
\hline & $62(16.7)$ & 29.44 & 26.90 & 2.00 & 28.16 & 26.29 & 2.23 & 26.81 & 26.81 & 2.48 & 25.62 & 25.62 & 2.75 \\
\hline & $57(13.9)$ & 29.10 & 29.10 & 2.00 & 28.01 & 28.01 & 2.23 & 26.85 & 26.85 & 2.48 & 25.62 & 25.62 & 2.75 \\
\hline \multirow{5}{*}{1125} & $72(22.2)$ & 35.27 & 18.78 & 2.06 & 33.67 & 18.21 & 2.28 & 31.96 & 17.61 & 2.53 & 30.22 & 17.01 & 2.81 \\
\hline & $67(19.4)$ & 32.36 & 23.68 & 2.05 & 30.87 & 23.10 & 2.28 & 29.29 & 22.50 & 2.53 & 27.66 & 21.88 & 2.80 \\
\hline & $63(17.2) \mathrm{tt}$ & 30.25 & 22.90 & 2.05 & 28.84 & 22.31 & 2.28 & 27.36 & 21.70 & 2.52 & 25.82 & 21.07 & 2.80 \\
\hline & $62(16.7)$ & 30.02 & 28.49 & 2.05 & 28.84 & 28.84 & 2.28 & 27.62 & 27.62 & 2.52 & 26.32 & 26.32 & 2.80 \\
\hline & $57(13.9)$ & 29.99 & 29.99 & 2.05 & 28.84 & 28.84 & 2.28 & 27.62 & 27.62 & 2.52 & 26.32 & 26.32 & 2.80 \\
\hline
\end{tabular}

\section{Simulation study:}

- 5 leading manufacturers ( $76 \%$ of the market)

- 450+ air-conditioners and heat pumps

- SEER 13-21 


\section{Results: Specific A/C Units}

\section{Houston: 2,500 ft' ${ }^{2}$ IECC 2009}

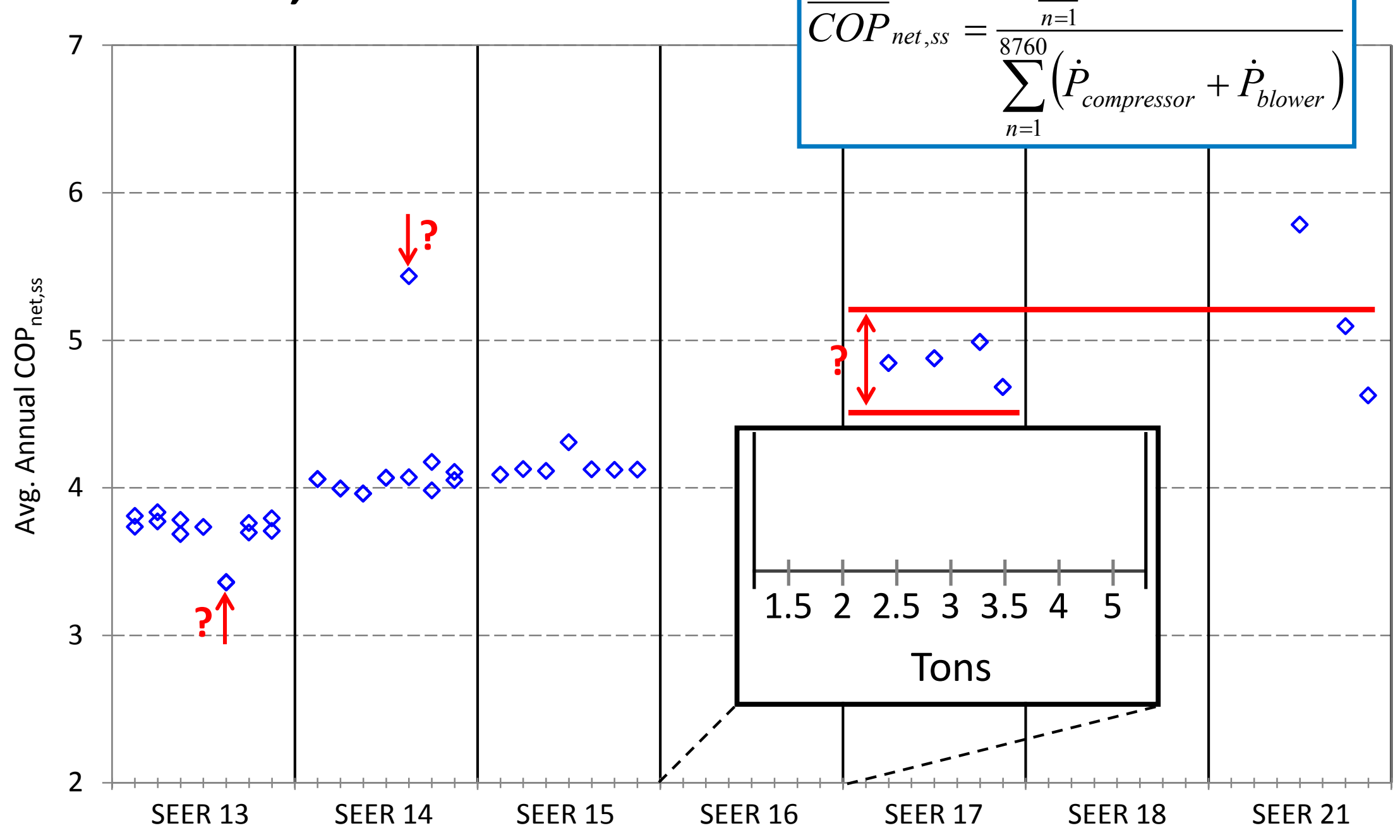




\section{Results: Specific A/C Units}

Houston: 2,500 ft' ${ }^{2}$ IECC 2009

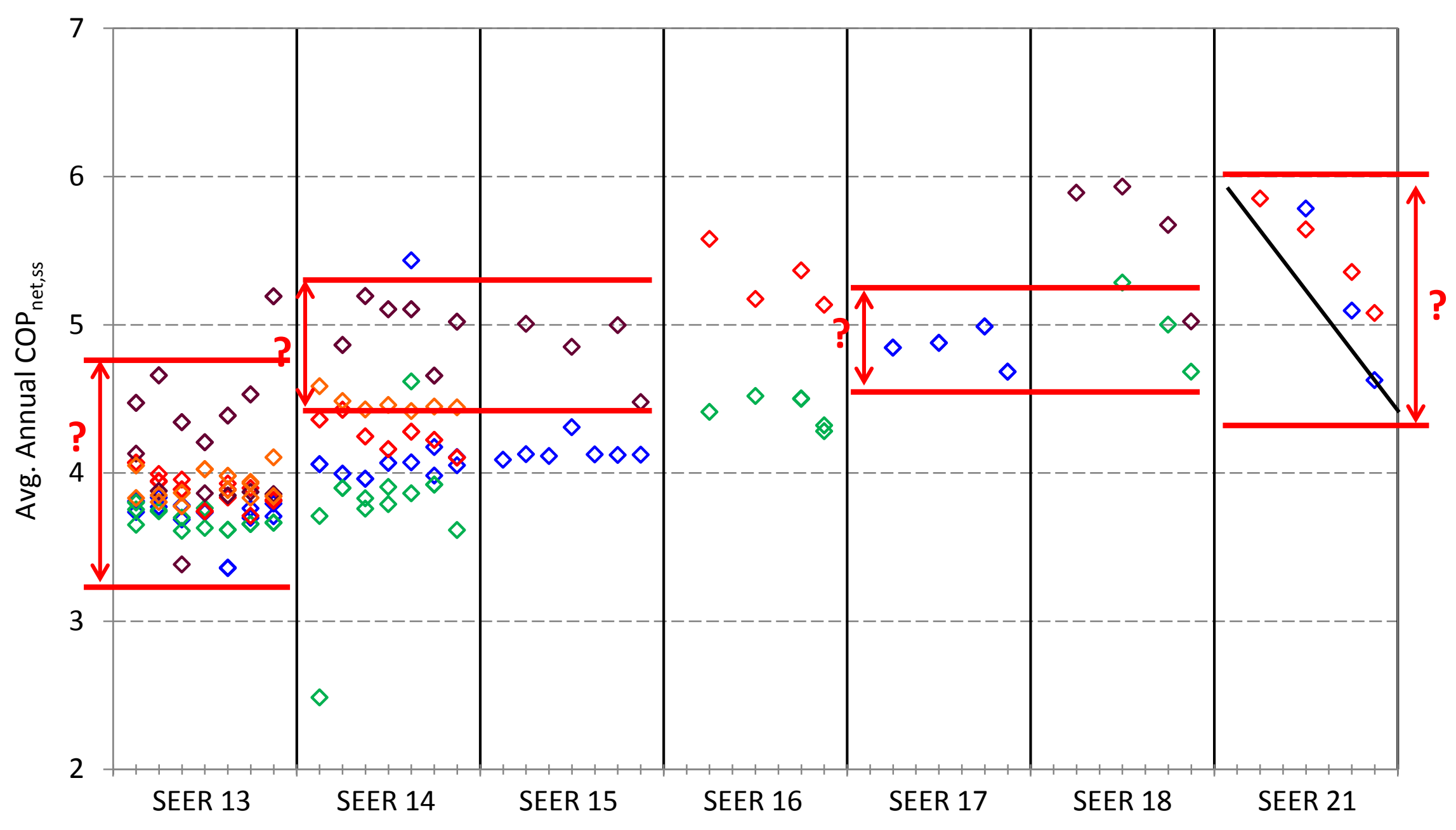




\section{Results: Specific A/C Units}

Question \#1: What leads to the variation in average annual COP?

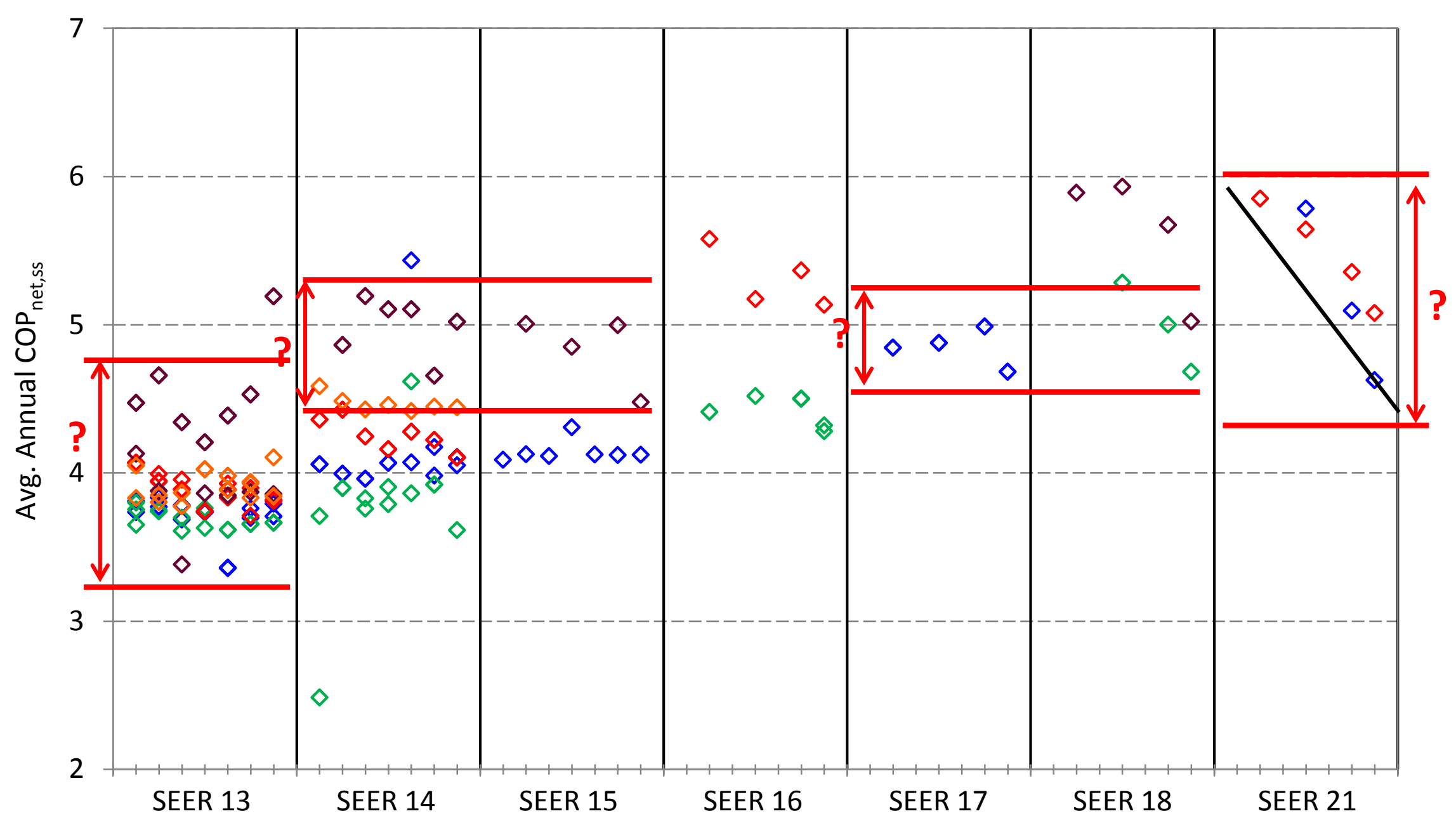




\section{Sensitivity to Curve Inputs}

- Simulation inputs

- Rated values (capacity, efficiency, etc.)

- Performance curves

- Which type of input is leading to the observed variation?

- What happens if a selected set of curves are used for all simulations? 


\section{Results: Sensitivity to Curve Inputs}

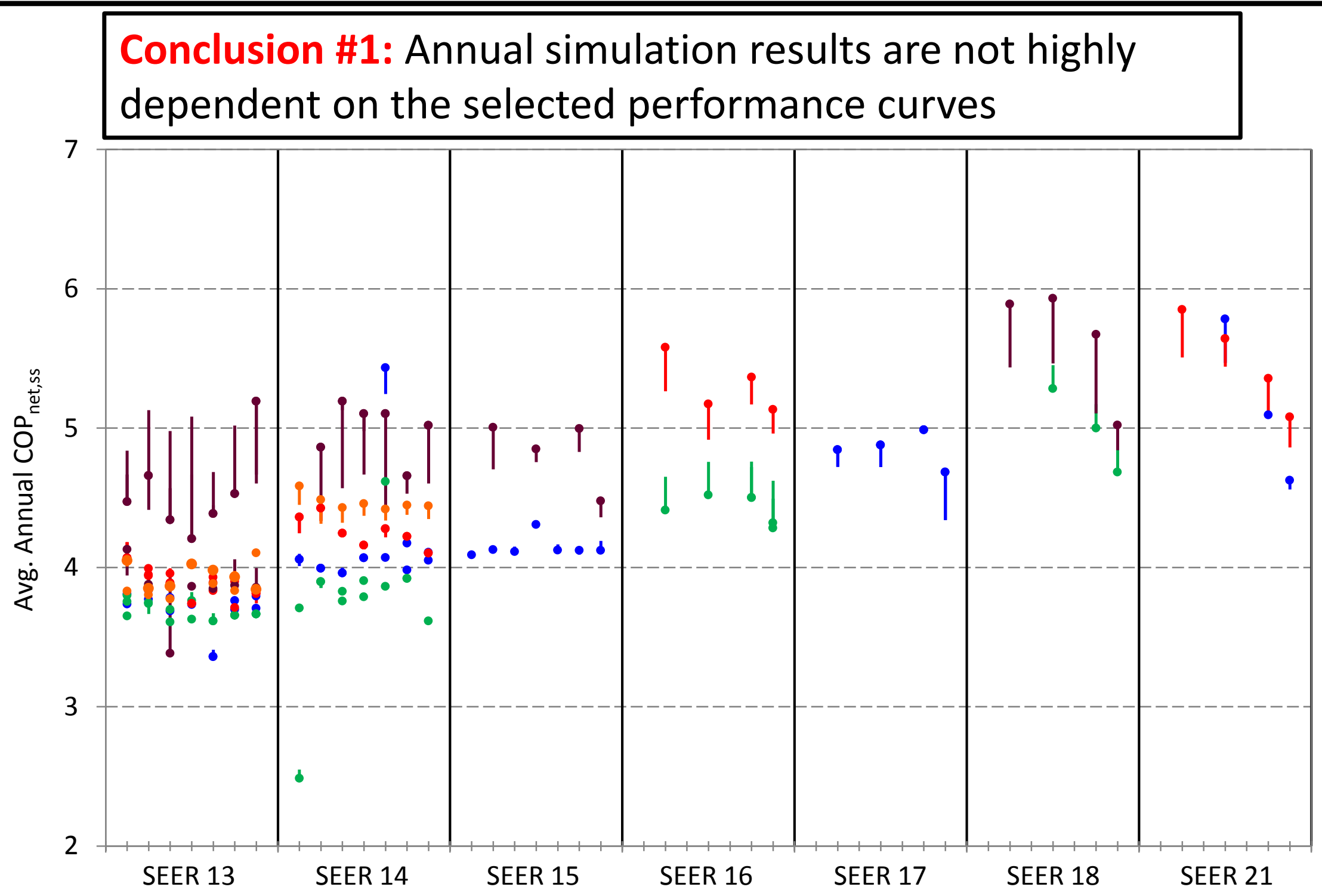




\section{Results: Specific A/C Units}

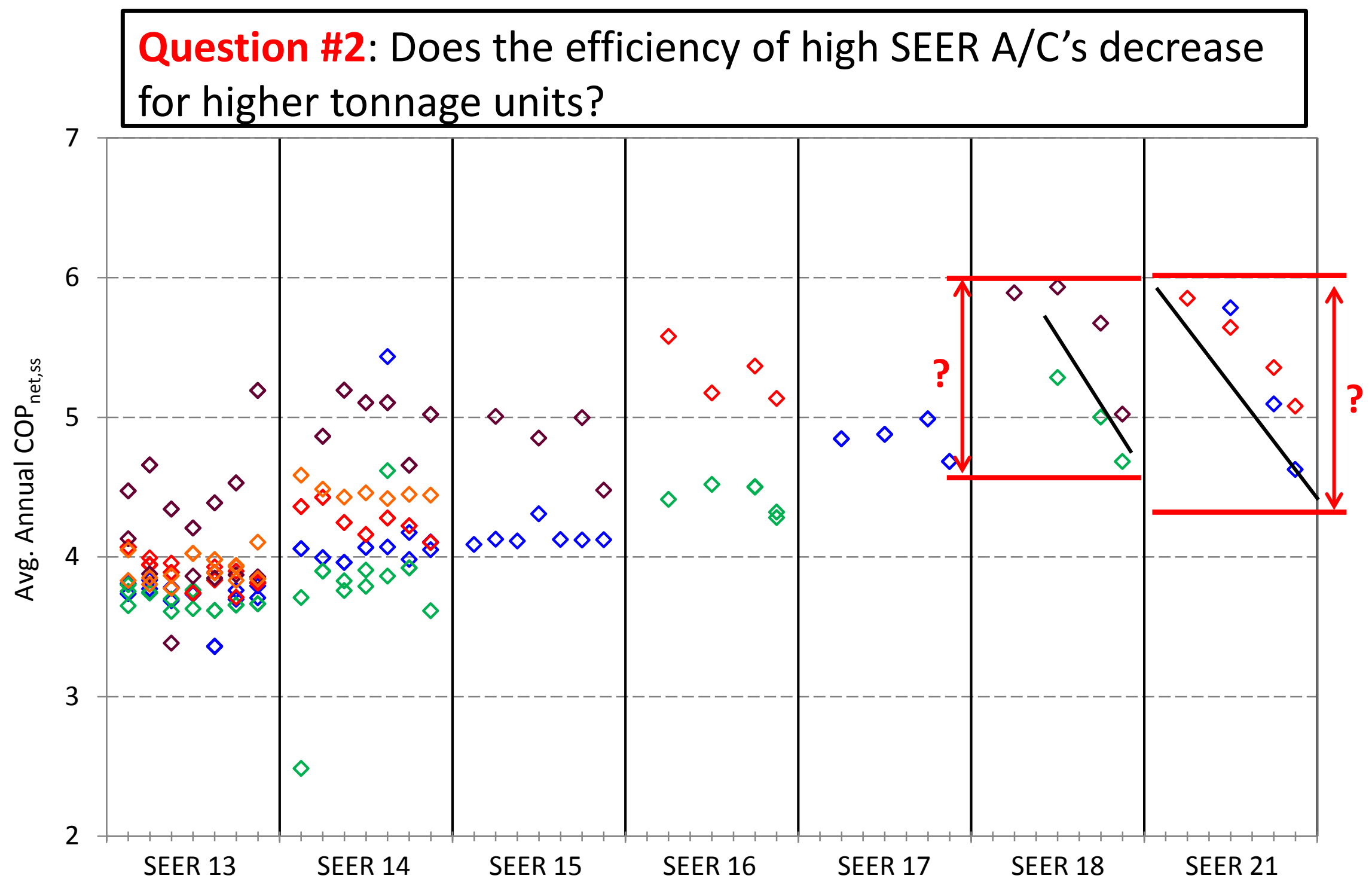




\section{Results: SEER vs. Tonnage}

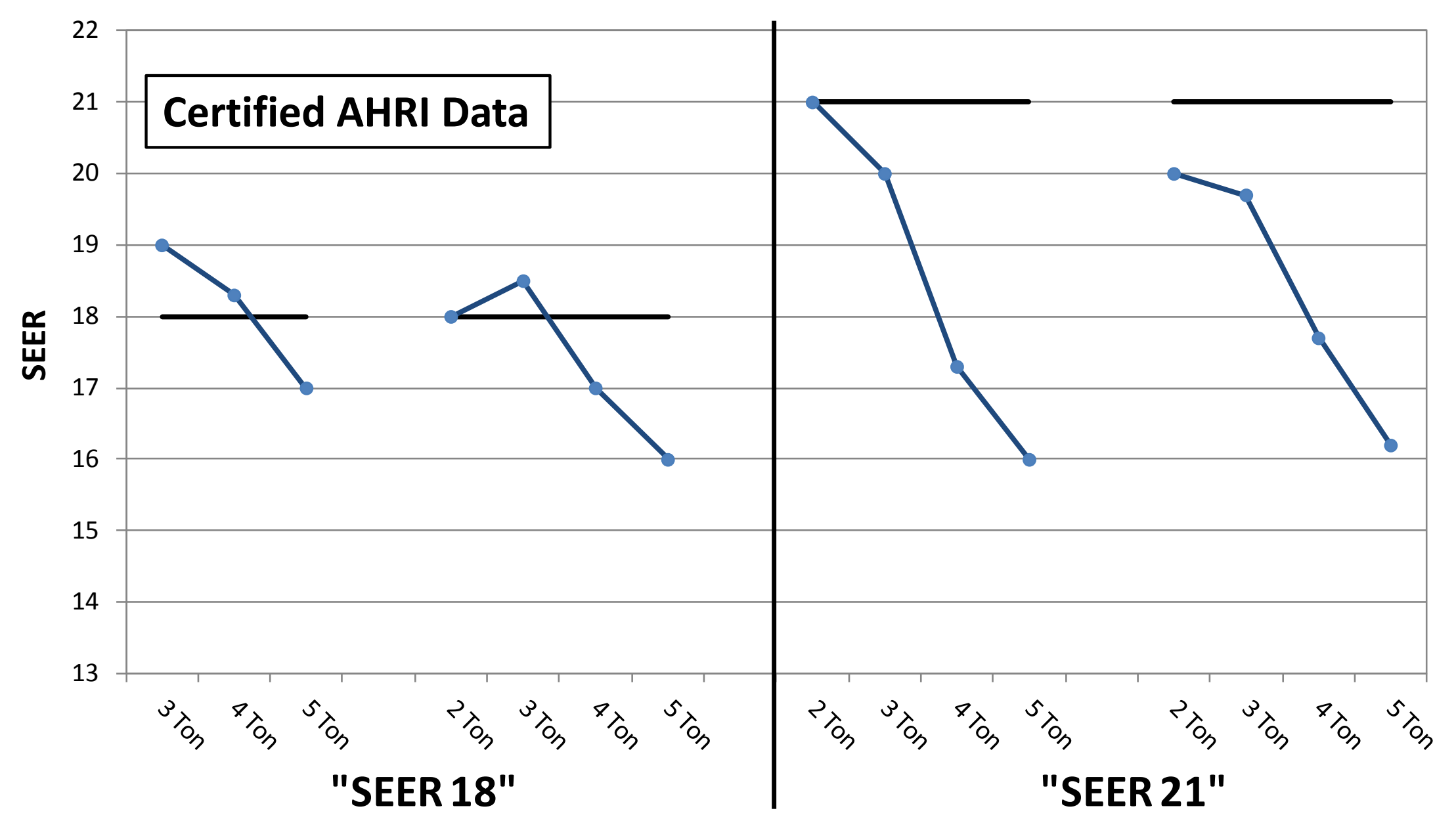

Conclusion \#2: SEER is highly effected by nominal tonnage for high efficiency equipment 


\section{Results: Specific A/C Units}

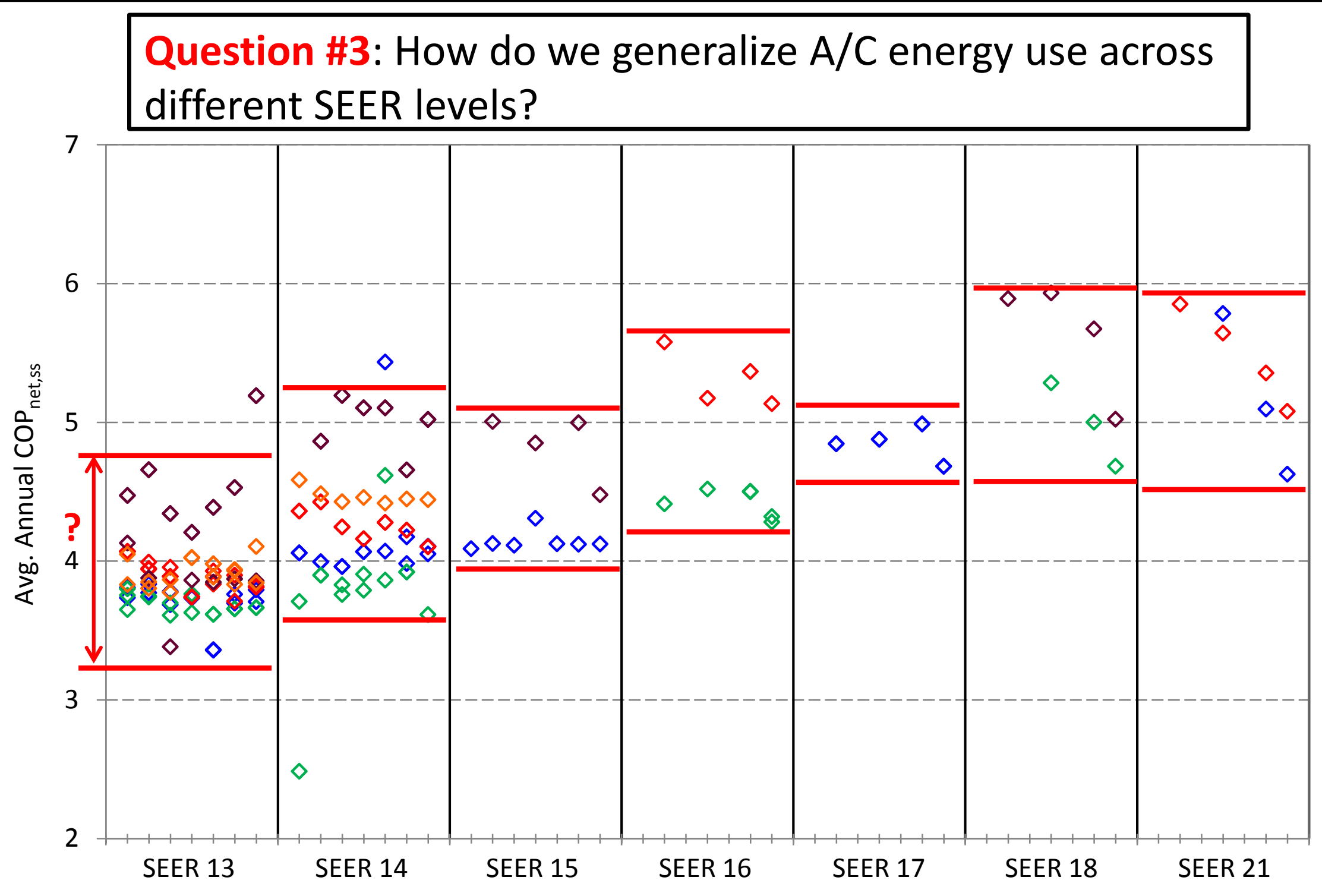




\section{Results: Standardized Set of A/C Options}

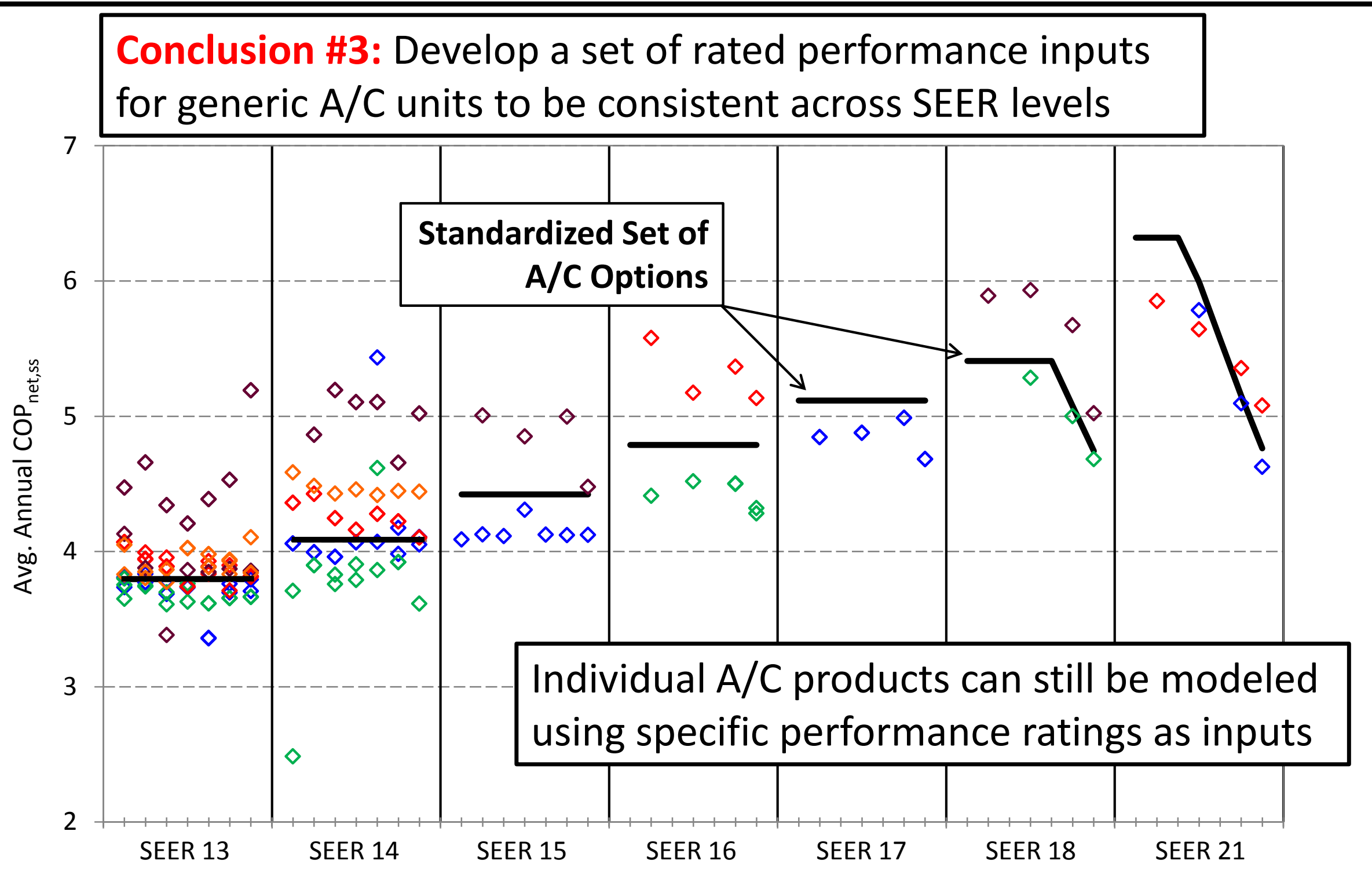




\section{Conclusions}

When comparing different $A / C$ 's:

1. Specific performance curves don't really matter

2. Be careful of "Up to SEER..."

3. The rated performance data does matter and needs to be consistent 


\section{Application: BEopt - Simplified Inputs}

\section{BEopt 1.1}

\begin{tabular}{ll}
\hline Cooling EER & 12.72 \\
COOL_CAP_FT_SPEC_coefficients & $1.61196622,-0.03412895,0.00044551,0.00278667,-0.00000211,-0.00008969$ \\
COOL_SH_FT_SPEC_coefficients & $0.42251614,0.07360185,-0.00089453,-0.00840292,0.00001615,0.00004937$ \\
COIL_BF_FT_SPEC_coefficients & $56.67437760,-1.66560721,0.01245741,0.0,0.0,0.0$ \\
COOL_EIR_FT_SPEC_coefficients & $-0.68032067,0.03410170,-0.00025020,0.00559185,0.00010461,-0.00015031$ \\
COOL_EIR_FPLR_SPEC_coefficients & $0.00000728,1.09852231,-0.11487027,0.01634067$ \\
COOL_CLOSS_FPLR_SPEC_coefficients & $0.90949556,0.09864773,-0.00819488$ \\
COIL_BF_FFLOW_SPEC_Max & 1.0 \\
COIL_BF_FFLOW_SPEC_Min & 0.0 \\
COIL_BF_FFLOW_SPEC_coefficients & $1.0,0.0$ \\
FAN_EIR_FPLR_SPEC_coefficients & $0.0,1.0,0.0,0.0$ \\
SupplyKW & 0.000256 \\
CoilBF & 0.100700 \\
\hline
\end{tabular}

\section{BEopt 1.2}

\begin{tabular}{ll}
\hline Cooling SEER & 14.0 \\
Cooling EER & 11.98 \\
SHR & 0.74 \\
SupplyFanPower & 0.365 \\
\hline
\end{tabular}

User inputs for single stage units

\section{- Benefits}

- Easily model specific make \& model A/C's

- Negligible sacrifice in accuracy/flexibility 


\section{Application: BEopt - Consistent Results}

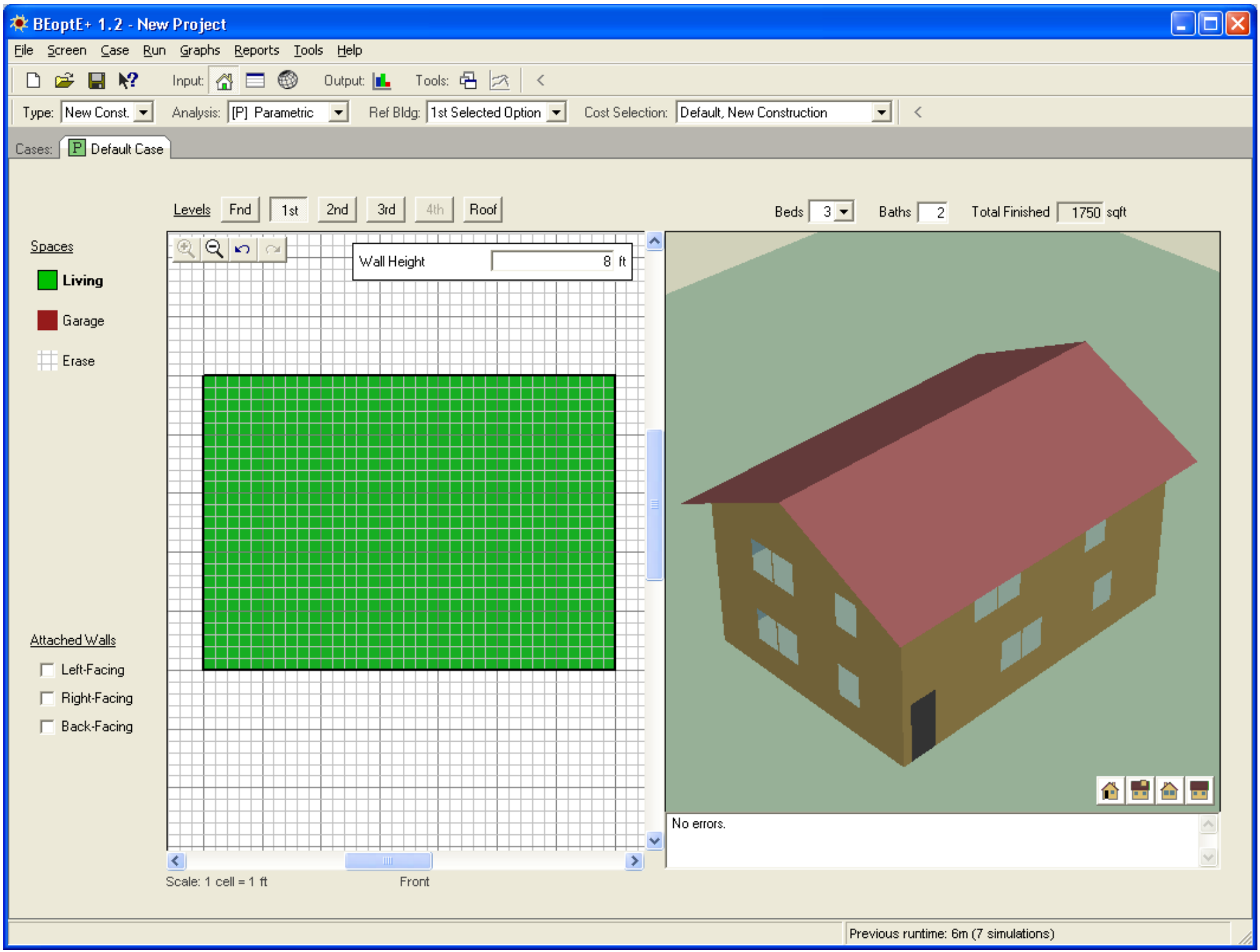




\section{Application: BEopt - Consistent Results}

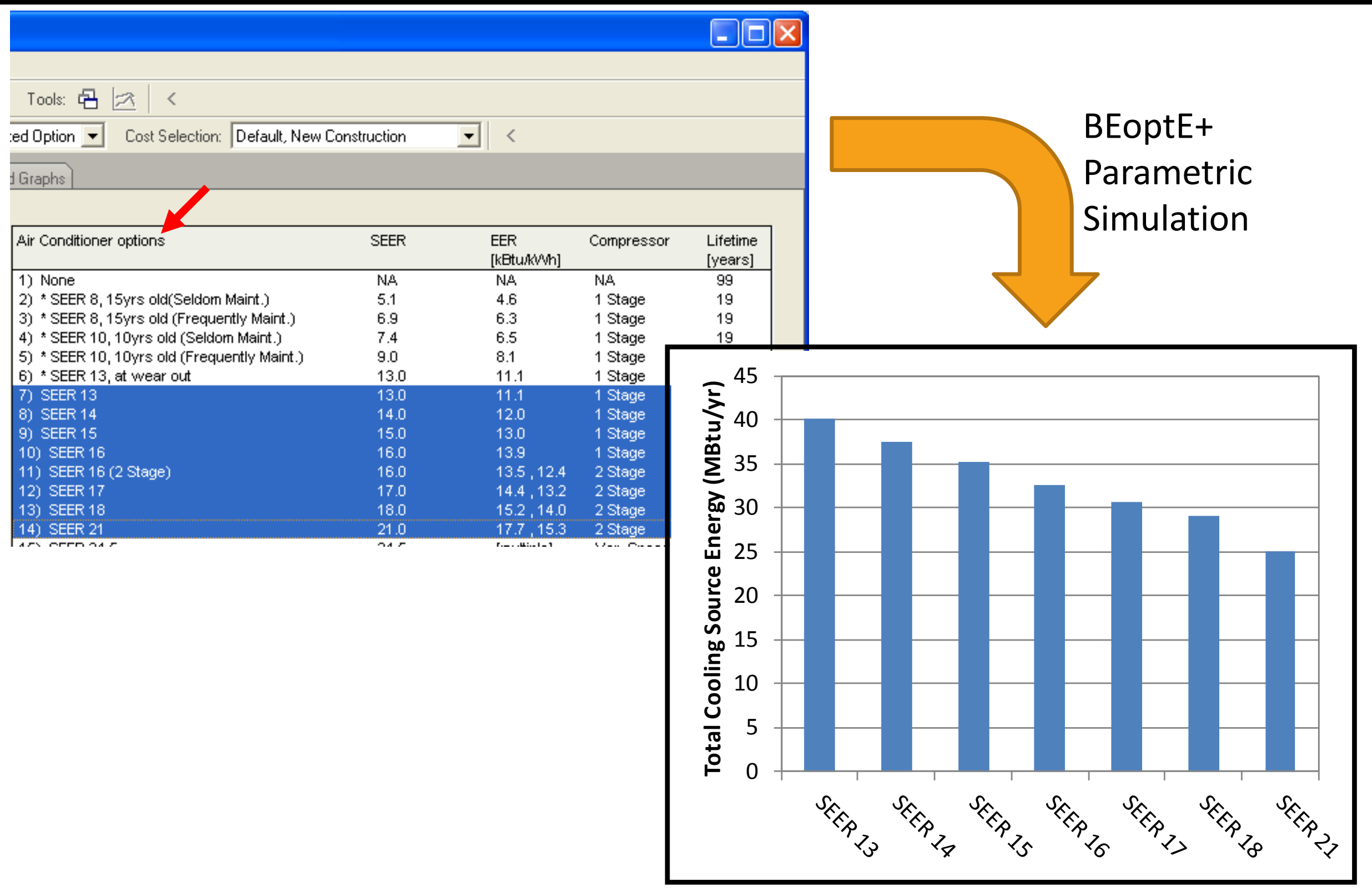




\section{Building America Gaps and Barriers}

- Gap: Analysis Methods and Tools Standing Technical Committee

- BA Strategic Plans: http://www1.eere.energy.gov/buildings/building america/strategic plan.html

- Achievements

- "... it is important to continually update and advance airconditioner and heat pump modeling approaches and capabilities."

- "Additionally, it is important that users can easily enter specific air-conditioners into building simulation software (such as BEopt) in order to compare specific manufactured units not included in built-in component libraries." 
